ings should be taken into consideration and evaluated thoroughly in better preclinical in vivo tumor models, if available. As rapid treatment resistance was shown with use of a Hh antagonist in a medulloblastoma patient (12), further research into the mechanisms of ATO action on GLI protein function may provide insights into the use of this agent either at the time of tumor relapse after Smo antagonist treatment or in combination with Smo antagonists upfront. In conclusion, as resistance to the current therapeutic modalities that are being used to treat cancer is a major hurdle in the clinic, future preclinical studies similar to those described by Beauchamp et al. (13) may lead to additional treatment strategies for cancer patients who relapse despite aggressive interventions.

\section{Acknowledgments}

The author was a Fellow of the Pediatric Scientist Development Program (NICHD $\mathrm{K} 12$-HD00850) and is currently funded by a Clinical Scientist Award (NINDS K08NS066083).

Address correspondence to: G. Praveen Raju, Department of Pediatrics/Child Neurology, Weill Cornell Medical College, 525
East 68th Street, Box 91, New York, New York 10065, USA. Phone: 212.746.1279; Fax: 212.746.4001; E-mail: prr9001@med. cornell.edu.

1. Emadi A, Gore SD. Arsenic trioxide - An old drug rediscovered. Blood Reviews. 2010;24(4-5):191-199.

2. Sun HD, Ma L, Hu XC, Zhang TD. Ai-Lin I treated 32 cases of acute promyelocytic leukemia. Chin J Integrated Tradit West Med. 1992;12:170-172.

3. Soignet SL, et al. United States multicenter study of arsenic trioxide in relapsed acute promyelocytic leukemia. J Clin Oncol. 2001;19(18):3852-3860.

4. Chen GQ, et al. In vitro studies on cellular and molecular mechanisms of arsenic trioxide $\left(\mathrm{As}_{2} \mathrm{O}_{3}\right)$ in the treatment of acute promyelocytic leukemia. Blood. 1996;88(3):1052-1061.

5. Porter AC, Fanger GR, Vaillancourt RR. Signal transduction pathways regulated by arsenate and arsenite. Oncogene. 1999;18(54):7794-7802.

6. Kapahi $\mathrm{P}$, et al. Inhibition of NF-kappa B activation by arsenite through reaction with a critical cysteine in the activation loop of Ikappa B kinase. J Biol Chem. 2000;275(46):36062-36066.

7. Jiang J, Hui CC. Hedgehog signaling in development and cancer. Dev Cell. 2008;15(6):801-812.

8. Teglund S, Toftgard R. Hedgehog beyond medulloblastoma and basal cell carcinoma. Biochim Biophys Acta. 2010;1805(2):181-208

9. Tremblay MR, McGovern K, Read MA, Castro AC. New developments in the discovery of small molecule Hedgehog antagonists. Curr Opin Chem Biol. 2010;14(3):428-435.

10. Taylor MD, et al. Mutations in SUFU predispose to medulloblastoma. Nat Genet. 2002;31(3):306-310.

11. Zwerner JP, et al. The EWS/FLI1 oncogenic transcription factor deregulates Gli1. Oncogene. 2008;27(23):3282-3291.
12. Rudin CM, et al. Treatment of medulloblastoma with hedgehog pathway inhibitor GDC-0449. N Engl J Med. 2009;361(12):1173-1178.

13. Beauchamp EM, et al. Arsenic trioxide inhibits human cancer cell growth and tumor development in mice by blocking Hedgehog/GLI pathway. J Clin Invest. 2011;121(1):148-160.

14. Beauchamp E, et al. GLI1 is a direct transcriptional target of EWS-FLI1 oncoprotein.J Biol Chem. 2009;284(1):9074.

15. Gore SD, et al. Single cycle of arsenic trioxide-based consolidation chemotherapy spares anthracycline exposure in the primary management of acute promyelocytic leukemia. JClin Oncol. 2010;28(6):1047-1053.

16. Au WY, Tam S, Fong BM, Kwong YL. Determinants of cerebrospinal fluid arsenic concentration in patients with acute promyelocytic leukemia on oral arsenic trioxide therapy. Blood. 2008;112(9):3587-3590.

17. Knipp S, Gattermann N, Schapira M, Kaferstein H, Germing U. Arsenic in the cerebrospinal fluid of a patient receiving arsenic trioxide for relapsed acute promyelocytic leukemia with CNS involvement. Leuk Res. 2007;31(11):1585-1587.

18. Kim J, Lee JJ, Kim J, Gardner D, Beachy PA. Arsenic antagonizes the Hedgehog pathway by preventing ciliary accumulation and reducing stability of the Gli2 transcriptional effector. Proc Natl Acad SciU S A. 2010;107(30):13432-13437.

19. Bai LY, et al. Differential expression of Sonichedgehog and Gli1 in hematological malignancies. Leukemia. 2008;22(1):226-228.

20. Lauth M, Bergstrom A, Shimokawa T, Toftgard R. Inhibition of GLI-mediated transcription and tumor cell growth by small molecule antagonists. Proc Natl Acad Sci U S A. 2007;104(20):8455-8460.

21. Kimura H, Ng JM, Curran T. Transient inhibition of the hedgehog pathway in young mice causes permanent defects in bone structure. Cancer Cell. $2008 ; 13(3): 249-260$

\title{
Gene defects in the soma: some get it and some don't!
}

\author{
Bernice Lo and Michael J. Lenardo
}

Laboratory of Immunology, NIAID, NIH, Bethesda, Maryland, USA.

\begin{abstract}
Advances in DNA sequencing technologies have increased attention on genetic variation in somatic tissues. Although long known to cause neoplastic diseases, somatic variation is now being investigated as a pathogenetic mechanism for other diseases. Somatic changes are genomic DNA variations that were not inherited but arise in tissues throughout life. In this issue of the JCI, Magerus-Chatinet et al. explore somatic changes in patients with autoimmune lymphoproliferative syndrome (ALPS), a congenital disease of defective apoptosis and autoimmunity that is usually associated with germline heterozygous mutations in the gene encoding the Fas death receptor. They explain why certain individuals have severe disease manifestations by documenting somatic alterations in the germline normal FAS allele in an unusual population of "double-negative" $T$ cells found in ALPS. Thus, the oncological concept of somatic loss of heterozygosity leading to selected cell expansion also applies to autoimmune diseases.
\end{abstract}

Conflict of interest: The authors have declared that no conflict of interest exists.

Citation for this article: J Clin Invest. 2011; 121(1):16-19. doi:10.1172/JCI45664.
The pathological role of somatic genetic changes in cancer has long been recognized, but the contribution of somatic mutations in other diseases is only now being uncovered. Somatic mutations are genetic alterations that are not inherited but arise in cells in the body over time. Recent reports have revealed an important role for somatic mutations in a variety of diseases, including ichthyosis with confetti, Wiskott-Aldrich syndrome, and severe combined immunodeficiency syndrome (1-3). Somatic mutations in highly proliferative tissues such as blood and epithelia may be more common than previously appreciated. These somatic events may only be evident when they provide the altered cells with a selective survival or proliferative advantage and cause clinical manifestations in the context of disease. In this issue of the JCI, Magerus-Chatinet and colleagues report on their discovery of how somatic mutations underlie the variable 

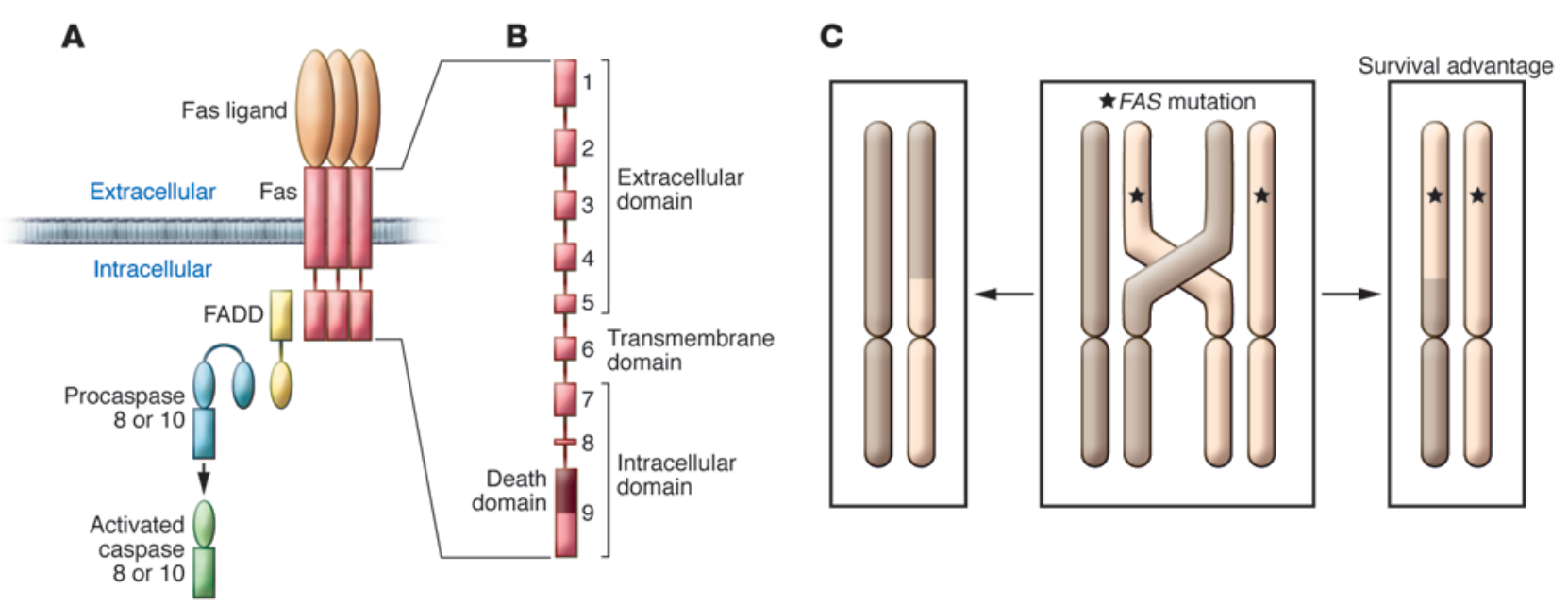

\section{Figure 1}

Fas receptor and its proximal signaling components. (A) Trimers of Fas ligand bind and oligomerize Fas receptor. Fas binds FAS-associated death domain protein (FADD), which recruits caspases 8 and 10, leading to their cleavage and activation. Mutations causing ALPS have been discovered in the genes encoding Fas, Fas ligand, and caspase 10. (B) FAS has 9 exons. Mutations in the portion of the gene encoding the intracellular domains, especially those affecting the death domain, often result in a more severe clinical disease with greater penetrance than those that affect the extracellular domains. (C) Mitotic recombination results in the somatic loss of the wild-type allele giving rise to cells homozygous for the FAS mutation that then have a survival advantage.

penetrance of clinical disease in certain cases of autoimmune lymphoproliferative syndrome (ALPS) (4).

\section{ALPS}

ALPS is an inherited disorder of lymphocyte apoptosis. Defective apoptosis results in chronic lymphoproliferation, characterized by lymphadenopathy and splenomegaly, autoimmunity, and the emergence of a normally rare population of TCR $\alpha \beta^{+}$T cells lacking CD4 or CD8 expression - so-called double-negative T cells (DNTs). The most common cause of ALPS is germline mutations in FAS. The second most common known cause of ALPS is somatic FAS mutations affecting primarily DNTs $(5,6)$. A smaller percentage of patients have germline mutations in genes encoding the Fas apoptosis signaling pathway components caspase 10 and Fas ligand (Figure 1). Approximately $20 \%$ of patients diagnosed with clinical ALPS have no known mutation.

ALPS is usually dominantly inherited; thus, most individuals inheriting a FAS mutation display manifestations of disease. The FAS mutations found in patients with ALPS often disrupt the signaling function of Fas by a dominant interfering mechanism. Since Fas trimerizes and forms higher-order oligomers required for signal transduction, FAS mutations typically encode defective proteins that enter complexes with the wild-type proteins and disrupt function (Figure 1A). Since prop- er trimer function requires complexes of exclusively wild-type Fas molecules, only one in eight trimers will be fully functional in heterozygote individuals. Defective Fas function makes lymphocytes from patients bearing ALPS-causing FAS mutations resistant to apoptosis.

Although ALPS is typically a dominantly inherited disease, patients carrying a homozygous FAS mutation develop earlyonset severe disease, whereas those with heterozygous FAS mutations can develop disease of variable intensity. The variation in disease severity is called variable "penetrance," and even individuals from the same family with the same heterozygous FAS mutations can range from having severe autoimmunity and lymphoproliferation to having no disease at all. Interestingly, $\mathrm{T}$ cells from ALPS patients and undiseased relatives harboring the same FAS mutation exhibit similar degrees of resistance to apoptosis. Hence, the apoptosis defect is completely penetrant, but the clinical phenotype is incompletely penetrant. Disease manifestations can also vary depending on the location of the mutation within the FAS gene. Heterozygous mutations that affect the extracellular domain of the Fas protein result in less clinical penetrance and milder disease than mutations affecting the intracellular portion of the protein (Figure 1B).

The cause of the variable penetrance of ALPS has been a long-standing question in the field. Is it the result of genet- ics or environment? If genetic, then how precisely does it work? Is there a modifier gene or additional genetic factor that is necessary for disease manifestation? In this issue of the JCI, Magerus-Chatinet and colleagues demonstrate that the "two-hit" or "loss of heterozygosity" concept, often used to describe the somatic genetic basis of cancer, applies to disease penetrance in patients with heterozygous germline FAS mutations (4). The two-hit hypothesis posits that in individuals with a heterozygous germline mutation, usually in reference to a tumor suppressor gene, a somatic second hit (or loss) affecting the wild-type allele is necessary to produce disease.

\section{Genetic mechanism for the variable penetrance of ALPS}

In their study (4), Magerus-Chatinet and colleagues examined seven ALPS patients and their asymptomatic relatives, all of whom bore heterozygous germline mutations affecting the extracellular domain of the Fas protein. They discovered that the seven affected individuals had a somatic "hit" in the DNTs that impaired or eliminated the wild-type FAS allele. Three of the individuals had somatic mutations in their second FAS allele, and four had telomeric uniparental disomy resulting in loss of the wild-type FAS allele and duplication of the mutant allele. Telomeric uniparental disomy is a rare event involving genetic recombination resulting in a cell having two cop- 
ies of a maternal or paternal chromosomal segment extending to the telomere and no copies of that segment from the other parent. These findings provide a striking new insight into a somatic genetic mechanism that can explain the onset of disease in ALPS patients carrying FAS mutations of incomplete penetrance. By exhibiting either compound heterozygous or homozygous FAS mutations, these patients effectively manifested homozygous recessive loss-offunction mutations, at least in their DNTs. Thus, more severe disease evidently emerges from a more severe loss of Fas function.

\section{The survival advantage of FAS mutations}

Mutations impairing apoptosis pathways give cells a survival advantage. Defective apoptosis has been found to be a key factor in the progression and development of cancers, especially those of hematopoietic origin (7). FAS mutations, in particular, result in the chronic premalignant lymphoproliferation characteristic of ALPS and an increased risk of lymphoma. Somatic mutations in FAS have also been found in various cases of leukemia, multiple myeloma, and lymphoma (8-10). Thus, it is no wonder that the same mechanisms often involved in malignant transformation also appear to be responsible for some cases of ALPS. The telomeric uniparental disomy described by Magerus-Chatinet et al. (4) is probably the result of mitotic recombination, a rare process of genetic alteration often linked to cancer in which maternal and paternal chromosomes in somatically dividing cells exchange segments of DNA. In the study by Magerus-Chatinet and colleagues (4), the healthy relatives carried only one copy of the FAS mutation, which apparently was insufficient to cause clinical disease on its own. Loss of heterozygosity in the affected ALPS patients due to telomeric uniparental disomy led to homozygosity of the FAS mutation (Figure 1C). These data suggest that two copies of the mutant allele, with loss of the wild-type allele, lead to clinical ALPS.

Three of the patients described by Magerus-Chatinet et al. (4) had a second hit in their other originally wild-type FAS allele. One of the somatic mutations was predicted to result in a severely truncated nonfunctional protein, and the other two mutations were in exon 9, which encodes the death domain that is critical for downstream signaling. Mutations in exon 9 often substantially impair Fas function $(11,12)$. Consequently, the second hit effectively removed any wild-type Fas protein. This unexpected genetic event presumably gave the mutant cells a greater survival advantage and promoted the onset of clinical disease.

\section{The role of DNTs as "drivers" of ALPS}

DNTs are a hallmark of ALPS. DNTs make up less than $2 \%$ of $\mathrm{T}$ cells in the blood of healthy individuals but can account for up to $40 \%$ in ALPS patients (13). Evidence suggests that the DNTs of ALPS patients may be derived from polyclonal chronically activated cytotoxic $T$ cells that have lost CD8 expression $(14,15)$. One unresolved question in the study of ALPS is whether DNTs are the "drivers" of disease or merely a manifestation of immune dysregulation. Previous findings that somatic FAS mutations in DNTs cause ALPS that is indistinguishable from germline FAS mutations suggest that the DNTs trigger the disease $(5,6)$. This report by Magerus-Chatinet and colleagues provides further evidence for the centrality of their role in the disease, since the secondary event correlating with clinical disease occurred mainly or perhaps wholly within the DNTs (4).

However, studies have shown that DNTs are hyporesponsive and do not proliferate, which would imply that they may not be the source of autoaggression $(14,16)$. According to this view, the DNTs represent a terminal state and perhaps the remaining footprint of effector $T$ cells that may have been the actual drivers of disease. Although ALPS-causing somatic mutations are predominantly detected in the DNTs, the somatic mutations may exist in single-positive $T$ cells but go undetected due to either the localization of these cells in lymphoid organs or to their small numbers compared with that of the unaffected T cells. In this scenario, DNTs are merely a sign or warning of the chronic lymphoproliferation that is occurring in vivo. Conceivably, there may not be a single cell subset that is the sole "driver" of disease. This is illustrated in a study by Stranges et al. (17), which demonstrated that a conditional knockout of FAS in T cells, B cells, or dendritic cells could result in systemic autoimmune disease. Further research is therefore necessary to clarify the contribution of DNTs and other cell subsets to the immunological derangements in ALPS.

\section{Concluding remarks}

The report by Magerus-Chatinet et al. (4) is one of the first to provide a molecular explanation for the variable penetrance seen in ALPS. The finding that a somatic mutation or loss of heterozygosity is a "modifier" of disease penetrance provides a new perspective on how autoimmune diseases may develop. Modifier alterations in other genes have been suspected for some time to be the basis of the variable penetrance seen in ALPS. The fact that the $l p r$ mouse, which is a model of ALPS, shows variable degrees of autoimmunity depending on the background mouse strain had long implied a role for modifier genes in disease $(18,19)$. The authors' discovery that a second hit in FAS itself predisposes to clinical ALPS (4) supports the concept that a modifier gene or genetic factor is responsible for disease expression but with a twist in that the "modifier" mutation is somatic and in the same gene. Future studies examining other ALPS families with incomplete penetrance may lead to the discovery of new modifier genes or a second hit in other genes involved in apoptosis or proliferation as well as other tolerance checkpoints. This two-hit model of disease expression may also help explain the onset of disease in other genetic disorders with selected effects on certain cell populations and incomplete penetrance.

\section{Acknowledgments}

This work was supported by the Division of Intramural Research, National Institute of Allergy and Infectious Diseases, NIH.

Address correspondence to: Michael Lenardo, Laboratory of Immunology, NIAID, NIH, Bldg. 10, Rm. 11N311, 10 Center Dr., MSC 1892, Bethesda, Maryland 208921892, USA. Phone: 301.496.6753; Fax: 301.402.8530; E-mail: lenardo@nih.gov.

1. Choate KA, et al. Mitotic recombination in patients with ichthyosis causes reversion of dominant mutations in KRT10. Science. 2010;330(6000):94-97.

2. Davis BR, Candotti F. Revertant somatic mosaicism in the Wiskott-Aldrich syndrome. Immunol Res. 2009;44(1-3):127-131.

3. Rieux-Laucat $F$, et al. Inherited and somatic CD3zeta mutations in a patient with T-cell deficiency. N Engl J Med. 2006;354(18):1913-1921.

4. Magerus-Chatinet A, et al. Onset of autoimmune lymphoproliferative syndrome (ALPS) in humans as a consequence of genetic defect accumulation. J Clin Invest. 2011;121(1):106-112.

5. Dowdell KC, et al. Somatic FAS mutations are common in patients with genetically undefined autoimmune lymphoproliferative syndrome. Blood. 2010;115(25):5164-5169.

6. Holzelova E, et al. Autoimmune lymphoproliferative syndrome with somatic Fas mutations. NEngl JMed. 2004;351(14):1409-1418.

7. Thompson CB. Apoptosis in the pathogenesis and treatment of disease. Science. 1995; 267(5203):1456-1462.

8. Landowski TH, Qu N, Buyuksal I, Painter JS, Dalton 
WS. Mutations in the Fas antigen in patients with multiple myeloma. Blood. 1997;90(11):4266-4270.

9. Tamiya S, Etoh K, Suzushima H, Takatsuki K, Matsuoka M. Mutation of CD95 (Fas/Apo-1) gene in adult T-cell leukemia cells. Blood. 1998; 91(10):3935-3942.

10. Beltinger C, Kurz E, Bohler T, Schrappe M, Ludwig WD, Debatin KM. CD95 (APO-1/Fas) mutations in childhood T-lineage acute lymphoblastic leukemia. Blood. 1998;91(10):3943-3951.

11. Fisher GH, et al. Dominant interfering Fas gene mutations impair apoptosis in a human autoimmune lymphoproliferative syndrome. Cell. 1995 ; 81(6):935-946.

12. Jackson CE, et al. Autoimmune lymphoproliferative syndrome with defective Fas: genotype influences penetrance. Am J Hum Genet. 1999; 64(4):1002-1014

13. Caminha I, et al. Using biomarkers to predict the presence of FAS mutations in patients with features of the autoimmune lymphoproliferative syndrome. J Allergy Clin Immunol. 2010; 125(4):946-949.

14. Bleesing JJ, et al. A composite picture of TcR alpha/ beta(+) CD4(-)CD8(-) T Cells (alpha/beta-DNTCs) in humans with autoimmune lymphoproliferative syndrome. Clin Immunol. 2002;104(1):21-30.

15. Landolfi MM, Van Houten N, Russell JQ, Scollay R, Parnes JR, Budd RC. CD2-CD4-CD8-lymph node T lymphocytes in MRL lpr/lpr mice are derived from a CD2+CD4+CD8+ thymic precursor. J Immunol. 1993;151(2):1086-1096.
16. Sobel ES, Kakkanaiah VN, Rapoport RG, Eisenberg RA, Cohen PL. The abnormal lpr double-negative T cell fails to proliferate in vivo. Clin Immunol Immunopathol. 1995;74(2):177-184.

17. Stranges PB, et al. Elimination of antigen-presenting cells and autoreactive $T$ cells by Fas contributes to prevention of autoimmunity. Immunity. 2007;26(5):629-641.

18. Izui S, Kelley VE, Masuda K, Yoshida H, Roths JB, Murphy ED. Induction of various autoantibodies by mutant gene lpr in several strains of mice. J Immunol. 1984;133(1):227-233.

19. Wang Y, Nose M, Kamoto T, Nishimura M, Hiai H. Host modifier genes affect mouse autoimmunity induced by the lpr gene. Am J Pathol. 1997; 151(6):1791-1798.

\title{
A stimulating way to improve $T$ cell responses to poxvirus-vectored vaccines
}

\author{
Stuart N. Isaacs
}

University of Pennsylvania School of Medicine and Philadelphia VA Medical Center, Philadelphia, Pennsylvania, USA.

\begin{abstract}
Vaccines remain one of the most cost-effective public health measures. Despite ongoing efforts, protective vaccines against cancer and many infectious diseases, including malaria, tuberculosis, and HIV/AIDS, are still not in hand. Most investigators believe that to succeed against these difficult targets, vaccines that generate potent $T$ cell responses are needed. In this issue of the JCI, Salek-Ardakani et al. show how the relative virulence of a virus/vaccine vector affects the memory $\mathrm{CD8}^{+} \mathrm{T}$ cells generated and how the response may be enhanced. The work has important implications for the development of future vaccines that aim to trigger $\mathrm{CD8}^{+} \mathrm{T}$ cell responses.
\end{abstract}

Disease prevention is the key to public health. One of the most cost-effective ways to prevent disease is through vaccination, an approach that has enabled successful control of many infectious diseases that were once common, including measles, diphtheria, and pertussis (whooping cough). In addition, a protective vaccine was key to the global eradication of smallpox, and it is believed that vaccinations may soon relegate polio to a historical memory. However, many infectious diseases, including malaria, tuberculosis, and HIV/AIDS, have proven to be formidable foes; despite intensive research efforts, we still do not have efficacious protective vaccines. Most believe that to be successful, such vaccines - as well as vaccines that protect against cancer - will need to generate potent $\mathrm{T}$ cell responses.

Poxviruses are members of a large family of DNA viruses that share a long his-

Conflict of interest: The author has declared that no conflict of interest exists.

Citation for this article: J Clin Invest. 2011; 121(1):19-21. doi:10.1172/JCI45726. tory with vaccination. Cowpox virus was the active agent in Jenner's pioneering approaches to preventing smallpox in the 18 th century and is closely related to vaccinia virus, which was used as the vaccine that helped eradicate smallpox in the latter half of the 20th century. Remarkably, vaccinia virus remains one of the leading vector candidates to be used in the development of vaccines against challenging infectious diseases and cancers, largely as a result of the promise seen in the earliest studies that showed its utility as an expression vector $(1,2)$ and its ability to prime $\mathrm{T}$ cell responses to expressed foreign antigens (3). However, the laboratory strains of vaccinia virus, and even the old conventional human smallpox vaccines, are likely not ideal vaccine vectors from a safety perspective, especially in current times, when greater numbers of immunocompromised people are likely to be vaccinated or inadvertently exposed; even in normal individuals, what is considered an acceptable risk/benefit ratio has shifted. This has led to the pursuit of more attenuated and replication-incompetent poxvirus-based vaccine vectors that have significantly improved safety profiles. A recent achievement that highlights the promise of poxvirus-based vaccines is the somewhat unexpected results of the Thai HIV vaccine trial (4). In that study, vaccination with a recombinant Canarypox virus vector expressing three HIV proteins along with boosts of HIV envelope proteins showed modest protection from HIV acquisition. While some worry about the reproducibility of the effect found, others are clearly encouraged by the results and are seeking to understand and improve upon the immune responses generated by the vaccination strategy. Since vaccineinduced sterilizing immunity (defined as antibody and innate immune responses that prevent actual infection) is a high bar to reach against the problematic organisms that cause malaria, tuberculosis, and HIV/AIDS, future vaccines will need to generate potent antibody and cytotoxic $T$ cell responses.

In this issue of the JCI, Salek-Ardakani et al. show how the relative virulence of a virus/vaccine affects the memory $\mathrm{CD}^{+}$ $\mathrm{T}$ cells that are generated and how one might manipulate costimulatory molecules to enhance the $\mathrm{CD} 8^{+} \mathrm{T}$ cell response (5). The work has important implications for development of future vaccines that aim to target the $\mathrm{CD}^{+} \mathrm{T}$ cell response and the stimulatory molecules that might be candidates to generate such responses. 